\title{
Orthonormal Bernstein polynomials for Volterra integral equa- tions of the second kind
}

\author{
Jafar Biazar*, Hamed Ebrahimi \\ Department of Applied Mathematics, Faculty of Mathematical Sciences, University of Guilan, P. O. Box. 41635-19141, P. C. \\ 4193833697, Rasht, Iran \\ *Corresponding authorE-mail: biazar@guilan.ac.ir
}

\begin{abstract}
The purpose of this research is to provide an effective numerical method for solving linear Volterra integral equations of the second kind. The mathematical modeling of many phenomena in various branches of sciences lead into an integral equation. The proposed approach is based on the method of moments (Galerkin- Ritz) using orthonormal Bernstein polynomials. To solve a Volterra integral equation, the approximation for a solution is considered as an expansion in terms of Bernstein orthonormal polynomials. Ultimately, the usefulness and extraordinary accuracy of the proposed approach will be verified by a few examples where the results are plotted in diagrams, Also the results and relative errors are presented in some Tables.
\end{abstract}

Keywords: Volterra integral equations, linear integral equations, Orthonormal Bernstein polynomials, Gram-Schmidt's Process, moments (GalerkinRitz)

\section{Introduction}

Basically, a Volterra integral equation is an initial values problem. We consider then the numerical solution of the following non-singular Volterra integral equation of the second kind

$$
u(s)=y(s)+\lambda \int_{0}^{s} k(s, t) u(t) d t, \quad 0 \leq s \leq 1,
$$

where $u(s)$ the unknown is function on $[0,1], \lambda$ is a parameter, $k(s, t)$ and $y(s)$ are analytical functions on $[0,1] \times[0,1]$ and $[0,1]$, respectively. $k(s, t)$ satisfies the Lipschitz condition in $t$; these conditions will ensure that a unique continuos solution to the problem (1) exists. The Volterra integral equations occur in many physical and biological problems.

Many different researchers, provide numerical solutions to (1) using one of the existing methods, i.e. least squares, or Galerkin, in which expansion coefficients are determined [1]. In general, these numerical methods transform the integral equation into a linear system of algebraic equations, which can be solved easily. The accuracy of the proposed numerical method of solution depends on the degree of the polynomial Bernstein. The desired accuracy can be achieved by increasing the polynomial degree only. On the one hand, the increase in the degree of computational costs increases, but on the other hand, the accuracy can be achieved, Other techniques do not have this feature and it has a fixed accuracy, Therefore, the proposed technique is better than the available techniques. The second part describes the basic concept of Bernstein polynomials and orthonormal Bernstein polynomials and some of the results. In the third section, the solving method is defined. In the fourth section, accuracy is shown with some numerical examples. A comparison of the exact answer with the numerical method and the relative error analysis is presented in this section. Finally, the fifth part contains the conclusion.

\section{Preliminary concepts}

In this section, some basic concepts and definitions associated with Bernstein polynomials are presented, then orthonormal Bernstein polynomials will be determined via the Gram- Schmidt's Process. About 100 years ago, Bernstein's polynomial was introduced by a Russian mathematician, Sergei Natanovinch Bernstein. The Bernstein polynomials are written as an expansion in terms of basis Bernstein 
polynomials. A stable numerical method for calculating these polynomials is the de casteljau algorithm. With the advent of computer graphics, Bernstein polynomials on [0,1] became more important in Bezier curves.

A function $u:[0,1] \rightarrow R$ when approximated by Bernstein's polynomial is defined as

$$
B_{n}(u ; s)=\sum_{k=0}^{n} \alpha B_{k, n}(s)
$$

So that $B_{k, n}(s)$ is defined as follows

$$
B_{k, n}(s)=\left(\begin{array}{l}
n \\
k
\end{array}\right) s^{k}(1-s)^{n-k}, \quad k=0, \ldots, n,
$$

Here $n$ is the degree of polynomial and $k$ is an index. Bernstein's polynomials are very effective in various mathematical fields and a sequence of linearly independent sentences that can be used as the basis for continuous functions in a vector space. Bernstein's polynomials were first used by Bernstein to prove the usefulness of Weierstrass Approximation theorem [2]. The Bernstein polynomials defined in (2) has the following properties, $[3,4]$.

$$
\begin{gathered}
B_{k, n}(s) \geq 0, \\
\sum_{k=0}^{n} B_{k, n}(s)=1, \\
B_{k, n}(1-s)=B_{n-k, n}(s), \quad k=0, \ldots, n, \\
B_{k, n}(s)=(1-s) B_{k, n-1}(s)+s B_{k-1, n-1}(s), \quad k=0, \ldots, n, \\
\sum_{i=0}^{n-k}(-1)^{i}\left(\begin{array}{l}
n \\
k
\end{array}\right)\left(\begin{array}{l}
n-k \\
i
\end{array}\right) s^{i+k}=\left(\begin{array}{l}
n \\
k
\end{array}\right) s^{k}(1-s)^{n-k}, \\
B_{k, n}(s)=\left(\begin{array}{l}
n \\
k
\end{array}\right) s^{k}(1-s)^{n-k}, \quad k=0, \ldots, n .
\end{gathered}
$$

\subsection{The Gram- Schmidt's Process for obtaining orthonormal Bernstein polynomials}

Schmidt's process: [5]. Let $\phi_{\circ}(s), \phi_{1}(s), \ldots$ be an arbitrary sequence of functions on an interval $(a, b)$, subject merely to conditions of integrability and linear independence. Throughout this chapter the finite interval $(a, b)$ may be replaced by an infinite intervals $(a, \infty)$ or $(-\infty, \infty)$, provided that all the integrals in the process exist. It is assumed that every linear combination $\psi=k_{\circ} \phi_{\circ}+\ldots+k_{m} \phi_{m}$ of a finite number of $\phi ' s$, with constant coefficients $k_{\circ}, \ldots, k_{m}$, not all zero,

Let

$$
d_{0}=\int_{a}^{b}\left[\phi_{0}(s)\right]^{2} d s, \quad g_{0}(s)=\frac{\phi_{0}(s)}{\sqrt{d_{0}}},
$$

So that $\int_{a}^{b}\left[g_{0}(s)\right]^{2} d s=1$. Let

$$
\begin{array}{rr}
G_{1}=\phi_{1}(s)-c_{10} g_{0}(s), & c_{10}=\int_{a}^{b} \phi_{1}(s) g_{0}(s) d s, \\
d_{1}=\int_{a}^{b}\left[G_{1}(s)\right]^{2} d s, & g_{1}(s)=\frac{G_{1}(s)}{\sqrt{d_{1}}} .
\end{array}
$$

Then

$$
\int_{a}^{b} G_{1} g_{0} d s=0=\int_{a}^{b} g_{1} g_{0} d s, \quad \quad \int_{a}^{b} g_{1}^{2} d s=1
$$

In general, let functions $g_{2}, g_{3}, \ldots$ be defined successively by the relations

$$
\begin{aligned}
& G_{n}(s)=\phi_{n}(s)-\sum_{k=0}^{n-1} c_{n k} g_{k}(s), \quad c_{n k}=\int_{a}^{b} \phi_{n}(s) g_{k}(s) d s, \\
& d_{n}=\int_{a}^{b}\left[G_{n}(s)\right]^{2} d s, \quad g_{n}(s)=\frac{G_{n}(s)}{\sqrt{d_{n}}} .
\end{aligned}
$$


We now consider the Bernstein polynomials introduced in (3) :

$$
B_{k, n}(s)=\left(\begin{array}{l}
n \\
k
\end{array}\right) s^{k}(1-s)^{n-k}, \quad k=0, \ldots, n,
$$

using the Schmidt's process, we find orthonormal Bernstein polynomials of some degrees. Notation $O B(t)$ is used for such polynomials,

First degrees,

$$
\begin{aligned}
& O B_{01}(t)=\sqrt{3}(1-t), \\
& O B_{11}(t)=(3 t-1) .
\end{aligned}
$$

Six degrees;

$$
\begin{aligned}
& O B_{06}(t)=\sqrt{13}(t-1)^{6} \\
& O B_{16}(t)=-\sqrt{11}(t-1)^{5}(13 t-1) \\
& O B_{26}(t)=3(t-1)^{4}\left(78 t^{2}-24 t+1\right) \\
& O B_{36}(t)=-\sqrt{7}(t-1)^{3}\left(286 t^{3}-198 t^{2}+33 t-1\right) \\
& O B_{46}(t)=\sqrt{5}(t-1)^{2}\left(715 t^{4}-880 t^{3}+330 t^{2}-40 t+1\right) \\
& O B_{56}(t)=-\sqrt{3}(t-1)\left(1287 t^{5}-2475 t^{4}+1650 t^{3}-450 t^{2}+45 t-1\right), \\
& O B_{66}(t)=\left(1716 t^{6}-4752 t^{5}+4950 t^{4}-2400 t^{3}+540 t^{2}-48 t+1\right) .
\end{aligned}
$$

The main coefficients of these polynomials are as the following.

$$
\begin{aligned}
& O B_{06}(t)=\sqrt{13}(t-1)^{6} \ldots, \\
& O B_{16}(t)=-\sqrt{11}(t-1)^{5} \ldots \\
& O B_{26}(t)=3(t-1)^{4} \ldots \\
& O B_{36}(t)=-\sqrt{7}(t-1)^{3} \ldots \\
& O B_{46}(t)=\sqrt{5}(t-1)^{2} \ldots \\
& O B_{56}(t)=-\sqrt{3}(t-1) \ldots \\
& O B_{66}(t)=1 \ldots
\end{aligned}
$$

General form of these coefficients can be recognized as follows.

$$
(\sqrt{2(n-k)+1})(1-t)^{n-k}, \quad k=0, \ldots, n,
$$

and the general form of orthonormal Bernstein polynomial is equivalent to [6]

$$
\begin{gathered}
O B_{k, n}(t)=(\sqrt{2(n-k)+1})(1-t)^{n-k} \sum_{i=0}^{k}(-1)^{i}\left(\begin{array}{c}
2 n+1-i \\
k-i
\end{array}\right)\left(\begin{array}{c}
k \\
i
\end{array}\right) t^{k-i}, \\
O B_{n}(u ; s)=\sum_{k=0}^{n} u_{k} \times O B_{k, n}(s) .
\end{gathered}
$$

Following [7], we can have the same result for orthonormal Bernstein. The next theorems are well-known result of the convergence rate.

Theorem 2.1. For all functions $u(s) \in C[\circ, 1]$, the sequence $\left\{O B_{n}\right\}_{n=0}^{\infty}$ converges to $u(s)$, where $O B_{n}$ is defined in (16) 
From Theorem 2.1 it follows that, for any $u(s) \in C[\circ, 1]$ and for any $\varepsilon>0$, there is an integer such that,

$$
\left\|\mathrm{OB}_{n}(\mathrm{~s})-\mathrm{u}(\mathrm{s})\right\|<\varepsilon .
$$

Satisfy $n>\frac{X}{\delta^{2} \varepsilon}$, where $X=\|u\|$. Therefore $u(s) \in C[\circ, 1]$.

Theorem 2.2. If $u$ is bounded and has the first and second derivatives at Some $s \in[\circ, 1]$ then

$$
\lim _{n \rightarrow \infty} n\left(O B_{n}(s)-u(s)\right)=\frac{s(1-s)}{2} u(s)
$$

if $u(s)^{\prime \prime} \neq 0$, the rate of convergence is precisely $\frac{1}{n}$. So an error bound is obtained

$$
\left\|\mathrm{OB}_{n}(\mathrm{~s})-\mathrm{u}(\mathrm{s})\right\|<\frac{1}{8 n}\left\|u(s)^{\prime \prime}\right\| .
$$

\section{Proposed approach}

In this section, we use orthonormal Bernstein polynomials for numerical solution to (1). In fact, by using the method of moments (Galerkin- Ritz), we find the approximate solution of linear Volterra integral equations of the second kind.

\subsection{Method of moments for Volterra integral equations of the second kind}

Consider the numerical solution of the Volterra integral equations of the second kind to (1), can be approximated by an expansion of orthonormal Bernstein polynomials (16). Using the (Galerkin-Ritz) method, expansion coefficients in (16) can be obtained.

Consider the integral equation (1) :

$$
u(s)=y(s)+\lambda \int_{0}^{s} k(s, t) u(t) d t, \quad 0 \leq s \leq 1
$$

Let us consider $O B_{k, n}(s)$, introduced in (15), and $u(s)=\sum_{k=0}^{n} u_{k} O B_{k, n}(s)$ is an approximate solution to the equation, and residual is as the following

$$
\left.R(s)=y(s)-\left(u(s)+\lambda \int_{0}^{s} k(s, t) u(t)\right) d t\right)
$$

Moreover let us consider the following linear operator.

$$
\begin{aligned}
& \left.l(u(s))=u(s)+\lambda \int_{0}^{s} k(s, t) u(t)\right) d t, \\
& 0=\left\langle O B_{i, n}(s), R(s)\right\rangle=\int_{a}^{b} O B_{i, n}(s)[(y(s)-l(u(s))] d s, \\
& \int_{a}^{b} O B_{i, n}(s)\left(\sum_{k=0}^{n} u_{k} O B_{k, n}(s)-\lambda \int_{0}^{s} k(s, t) \sum_{k=0}^{n} u_{k} O B_{k, n}(t) d t\right) d s=\int_{a}^{b} O B_{i, n}(s) y(s) d s, \\
& \sum_{k=0}^{n} u_{k}\left(\int_{a}^{b} O B_{i, n}(s)\left(O B_{k, n}(s)-\lambda \int_{0}^{s} k(s, t) O B_{k, n}(t) d t\right) d s=\int_{a}^{b} O B_{i, n}(s) y(s),\right.
\end{aligned}
$$




$$
\begin{aligned}
& L_{i, k}(s)=\int_{a}^{b} O B_{i, n}(s) l_{k, n}(u(s)) d s, \\
& y_{i}(s)=\int_{a}^{b} O B_{i, n}(s) y(s) d s, \\
& L_{i, k}^{n+1}(s) u^{n+1}=y_{i}^{n+1} .
\end{aligned}
$$

Having the vector $u$, based on (21), the expansion coefficients are derived. Then we can find an approximate solution based on orthonormal Bernstein polynomials.

\section{Numerical examples}

In this section, we will be prove that the approximate solution obtained from the proposed method converges to the exact solution of the integral equations (1), a comparison of the exact solution and the approximate solution obtained from the numerical method is presented to demonstrate the efficiency of this method, so that $u(s)$ is an exact solution, and $O B_{n}$ is the approximate solution obtained using the othonormal Bernstein polynomials of degree $n$, as a basis.

\subsection{Relative error}

$$
e_{n}(s)=\frac{\left|u(s)-O B_{n}(s)\right|}{u(s)}
$$

(22) is the relative error obtained using a polynomial of degree $n$, so that $u(s)$ is an exact solution, and $O B_{n}$ is the approximate solution obtained on the basis of othonormal Bernstein polynomials of degree $n$. Computations are performed by Math Lab.

Example 1. Consider the following linear Volterra integral equation

$$
\mathrm{u}(\mathrm{s})=\mathrm{s}+1+\int_{0}^{s}(s-t) u(t) d t
$$

\begin{tabular}{|c|c|c|c|c|}
\hline points & Exact solutions & approx & & \\
\hline$s$ & $u(s)$ & $O B_{1}(s)$ & $O B_{2}(s)$ & $O B_{3}(s)$ \\
\hline 0.0 & 1.000000000000000 & 0.87111111111111 & 1.013085427968032 & 0.999056281195290 \\
\hline 0.1 & 1.105170918075648 & 1.040000000000000 & 1.106573771170649 & 1.105379332090889 \\
\hline 0.2 & 1.221402758160170 & 1.208888888888889 & 1.216845418029732 & 1.221798663434117 \\
\hline 0.3 & 1.349858807576003 & 1.377777777777778 & 1.343900368545281 & 1.349986009394116 \\
\hline 0.4 & 1.491824697641270 & 1.546666666666667 & 1.487738622717297 & 1.491613104140031 \\
\hline 0.5 & 1.648721270700128 & 1.715555555555556 & 1.648360180545778 & 1.648351681841001 \\
\hline 0.6 & 1.822118800390509 & 1.884444444444444 & 1.825765042030726 & 1.821873476666172 \\
\hline 0.7 & 2.013752707470477 & 2.053333333333333 & 2.019953207172140 & 2.013850222784684 \\
\hline
\end{tabular}

\section{with exact solution $u(s)=e^{s}$.}

The numerical results of this example by the proposed method are shown in Table 1 and Table 2 .

TABLE 1. Numerical results of Example 1. 


\begin{tabular}{|c|c|c|c|c|}
\hline 0.8 & 2.225540928492468 & 2.222222222222222 & 2.230924675970019 & 2.225953654365680 \\
\hline 0.9 & 2.459603111156950 & 2.391111111111111 & 2.458679448424365 & 2.459855505578303 \\
\hline 1 & 2.718281828459046 & 2.560000000000000 & 2.703217524535178 & 2.717227510591695 \\
\hline
\end{tabular}

\begin{tabular}{|c|c|c|c|c|}
\hline points & Exact solutions & $O B_{4}(s)$ & $O B_{5}(s)$ & $O B_{6}(s)$ \\
\hline$s$ & $u(s)$ & 1.000052775692482 & 0.999997589713689 & 1.000000093033118 \\
\hline 0.0 & 1.000000000000000 & 1.105149894378666 & 1.105171875173351 & 1.105170895086828 \\
\hline 0.1 & 1.105170918075648 & 1.221393908802883 & 1.221402360142629 & 1.221402788433854 \\
\hline 0.2 & 1.221402758160170 & 1.349986009394116 & 1.349858076251571 & 1.349858807043106 \\
\hline 0.3 & 1.349858807576003 & 1.491841759973453 & 1.491824870001362 & 1.491824669644988 \\
\hline 0.4 & 1.491824697641270 & 1.648722049216913 & 1.648722049133015 & 1.648721269827206 \\
\hline 0.5 & 1.648721270700128 & 1.822102139192618 & 1.822119028996073 & 1.822118828639727 \\
\hline 0.6 & 1.822118800390509 & 2.013737141092205 & 2.013751978917304 & 2.013752709708897 \\
\hline 0.7 & 2.013752707470477 & 2.225548920084550 & 2.225540468569400 & 2.225540896860714 \\
\hline 0.8 & 2.225540928492468 & 2.459626095315757 & 2.459604114339673 & 2.459603134253269 \\
\hline 0.9 & 2.459603111156950 & & 2.718279225698752 & 2.718281729018333 \\
\hline 1 & 2.718281828459046 & 2.718224039909170 & & \\
\hline
\end{tabular}

\begin{tabular}{|c|c|c|}
\hline points & Exact solutions & approximate solutions \\
\hline$s$ & $u(s)$ & $O B_{7}(s)$ \\
\hline 0.0 & 1.000000000000000 & 0.999999996890340 \\
\hline 0.1 & 1.105170918075648 & 1.105170918147056 \\
\hline 0.2 & 1.221402758160170 & 1.221402757476811 \\
\hline 0.3 & 1.349858807576003 & 1.349858808411033 \\
\hline 0.4 & 1.491824697641270 & 1.491824697790828 \\
\hline 0.5 & 1.648721270700128 & 1.648721269827206 \\
\hline 0.6 & 1.822118800390509 & 1.822118800493887 \\
\hline 0.7 & 2.013752707470477 & 2.013752708340969 \\
\hline 0.8 & 2.225540928492468 & 2.225540927817757 \\
\hline 0.9 & 2.459603111156950 & 2.459603111193040 \\
\hline 1 & 2.718281828459046 & 2.718281825161111 \\
\hline
\end{tabular}


TABLE 2. Relative errors of the results for Example 1.

\begin{tabular}{|c|c|c|c|c|}
\hline Points & & & errors & \\
\hline$S$ & $e_{1}(s)$ & $e_{2}(s)$ & $e_{3}(s)$ & $e_{4}(s)$ \\
\hline 0.0 & 0.128888888888889 & 0.013085427968032 & $0.943718804710003 \times 10^{-3}$ & $0.527756924819656 \times 10^{-4}$ \\
\hline 0.1 & 0.058969085242602 & 0.001269353972364 & $0.188580799433142 \times 10^{-3}$ & $0.190230276947461 \times 10^{-4}$ \\
\hline 0.2 & 0.010245489612395 & 0.003731234517026 & $0.324139823086040 \times 10^{-3}$ & $0.072452409559868 \times 10^{-4}$ \\
\hline 0.3 & 0.020682881828145 & 0.004414120200780 & $0.094233424562025 \times 10^{-3}$ & $0.942334245620251 \times 10^{-4}$ \\
\hline 0.4 & 0.036761671201789 & 0.002738977931143 & $0.141835365491372 \times 10^{-3}$ & $0.114372232944687 \times 10^{-4}$ \\
\hline 0.5 & 0.040537042884785 & 0.000219012249534 & $0.224166974548787 \times 10^{-3}$ & $0.004721942992266 \times 10^{-4}$ \\
\hline 0.6 & 0.034205038683854 & 0.002001099840162 & $0.134636514526160 \times 10^{-3}$ & $0.091438592739126 \times 10^{-4}$ \\
\hline 0.7 & 0.019655157118360 & 0.003079077028009 & $0.048424671929846 \times 10^{-3}$ & $0.077300346832117 \times 10^{-4}$ \\
\hline 0.8 & 0.001491190850619 & 0.002419073677157 & $0.185449689074751 \times 10^{-3}$ & $0.035908537919929 \times 10^{-4}$ \\
\hline 0.9 & 0.027846769153590 & 0.000375533242902 & $0.102615914009936 \times 10^{-3}$ & $0.093446616257096 \times 10^{-4}$ \\
\hline 1 & 0.058228630601108 & 0.005541847709149 & $0.387861867858151 \times 10^{-3}$ & $0.212592194345044 \times 10^{-4}$ \\
\hline
\end{tabular}

\begin{tabular}{|c|c|c|c|}
\hline \multicolumn{2}{|c|}{ Points } & \multicolumn{2}{|c|}{ elative errors } \\
\hline$s$ & $e_{5}(s)$ & $e_{6}(s)$ & $e_{7}(s)$ \\
\hline 0.0 & $0.241028631098317 \times 10^{-5}$ & $0.930331180981625 \times 10^{-7}$ & $0.310965997396551 \times 10^{-8}$ \\
\hline 0.1 & $0.086601781447345 \times 10^{-5}$ & $0.208011444951862 \times 10^{-7}$ & $0.006461262187028 \times 10^{-8}$ \\
\hline 0.2 & $0.032586920120216 \times 10^{-5}$ & $0.247859961031222 \times 10^{-7}$ & $0.055948714908432 \times 10^{-8}$ \\
\hline 0.3 & $0.054177846438258 \times 10^{-5}$ & $0.003947798516571 \times 10^{-7}$ & $0.061860547223407 \times 10^{-8}$ \\
\hline 0.4 & $0.011553642493740 \times 10^{-5}$ & $0.187664690118349 \times 10^{-7}$ & $0.010025182173463 \times 10^{-8}$ \\
\hline 0.5 & $0.047214341251753 \times 10^{-5}$ & $0.005294539304207 \times 10^{-7}$ & $0.052945393042066 \times 10^{-8}$ \\
\hline 0.6 & $0.012546139349604 \times 10^{-5}$ & $0.155034994687350 \times 10^{-7}$ & $0.005673503441521 \times 10^{-8}$ \\
\hline 0.7 & $0.036178879869362 \times 10^{-5}$ & $0.011115665332818 \times 10^{-7}$ & $0.043227359526289 \times 10^{-8}$ \\
\hline 0.8 & $0.020665675575159 \times 10^{-5}$ & $0.142130631056329 \times 10^{-7}$ & $0.030316717393788 \times 10^{-8}$ \\
\hline 0.9 & $0.040786365839404 \times 10^{-5}$ & $0.093902625894066 \times 10^{-7}$ & $0.001467319737172 \times 10^{-8}$ \\
\hline
\end{tabular}




\begin{tabular}{|c|c|c|c|}
\hline 1 & $0.095750200244416 \times 10^{-5}$ & $0.365821939271286 \times 10^{-7}$ & $0.121324241644133 \times 10^{-8}$ \\
\hline
\end{tabular}

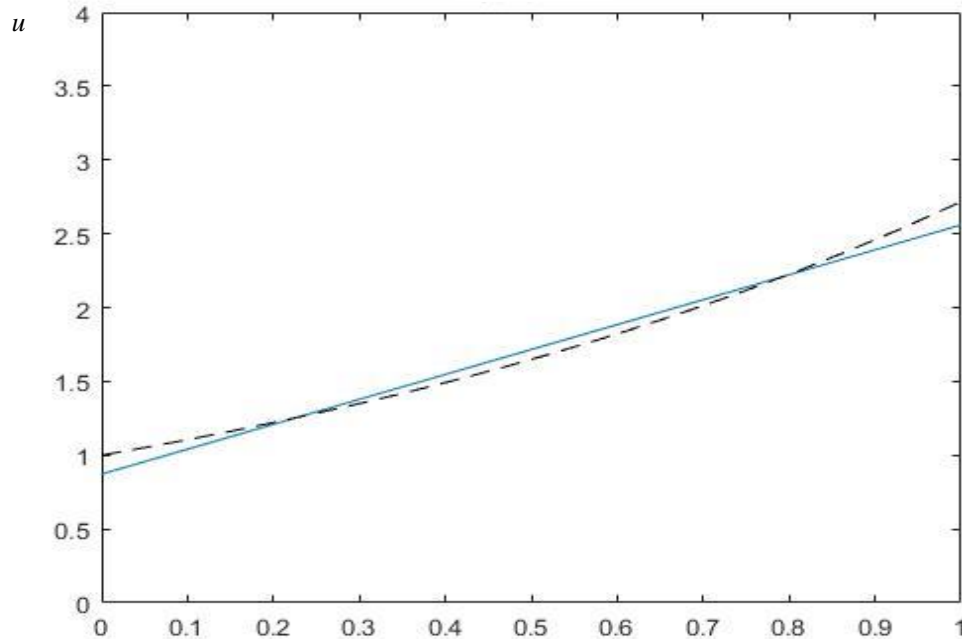

FIG. 1. Exact and approximate solutions of Example 1, for $n=1$.

Exact solution - - - .

Approximate solution

$u$

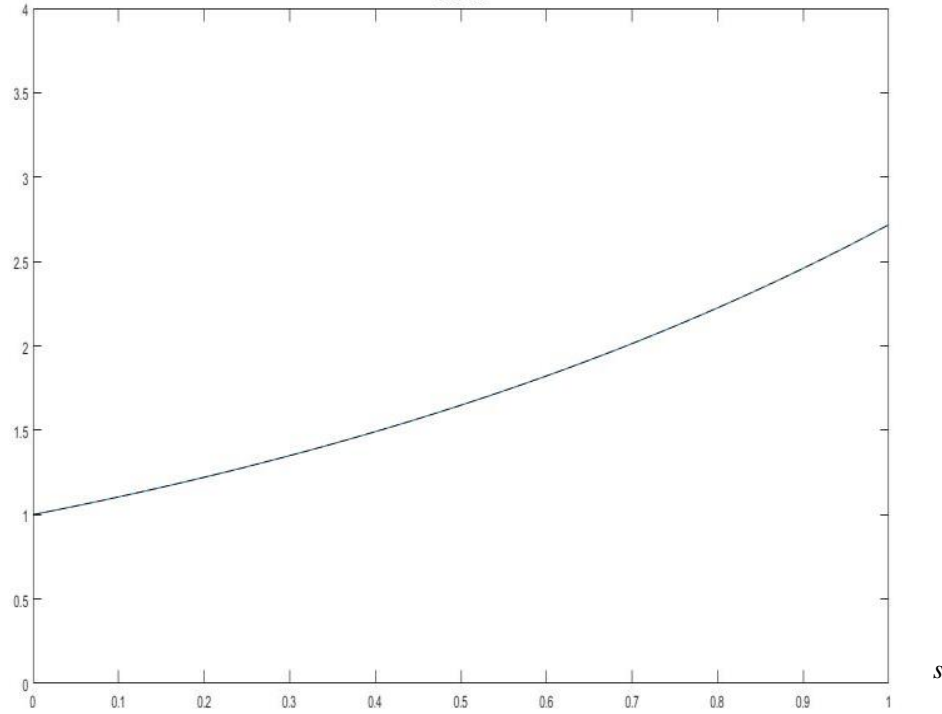

FIG. 2. Exact and approximate solutions of Example 1, for $n=7$.

Exact solution - - - -

Approximate solution

Example 2. Consider the following linear Volterra integral equation [8]

$\mathrm{u}(\mathrm{s})=\cosh (s)-\int_{0}^{s} e^{-(t-s)} u(t) d t$,

with exact solution $\mathrm{u}(\mathrm{s})=e^{-s}$.

The numerical results of this example by the proposed method are shown in Table 3 and Table 4 . 
TABLE 3. Numerical results of Example 2.

\begin{tabular}{|c|c|c|c|c|}
\hline points & Exact solutions & approximat & ons & \\
\hline$S$ & $u(s)$ & $O B_{1}(s)$ & $O B_{2}(s)$ & $O B_{3}(s)$ \\
\hline 0.0 & 1.000000000000000 & 0.948180838242837 & 0.994854691128702 & 0.999633926955816 \\
\hline 0.1 & 0.904837418035960 & 0.884968782359981 & 0.904689349671238 & 0.904932580780617 \\
\hline 0.2 & 0.818730753077982 & 0.821756726477125 & 0.820742307624633 & 0.818875353509198 \\
\hline 0.3 & 0.740818220681718 & 0.758544670594269 & 0.743013564988887 & 0.740844808077004 \\
\hline 0.4 & 0.670320046035639 & 0.695332614711414 & 0.671503121764002 & 0.670223507419479 \\
\hline 0.5 & 0.606530659712633 & 0.632120558828558 & 0.606210977949976 & 0.606394014472068 \\
\hline 0.6 & 0.548811636094027 & 0.568908502945702 & 0.547137133546810 & 0.548738892170214 \\
\hline 0.7 & 0.496585303791410 & 0.505696447062846 & 0.494281588554503 & 0.496640703449362 \\
\hline 0.8 & 0.449328964117222 & 0.442484391179990 & 0.447644342973056 & 0.449482011244956 \\
\hline 0.9 & 0.406569659740599 & 0.379272335297135 & 0.407225396802468 & 0.406645378492440 \\
\hline 1 & 0.367879441171442 & 0.316060279414279 & 0.373024750042740 & 0.367513368127258 \\
\hline
\end{tabular}

\begin{tabular}{|c|c|c|c|c|}
\hline points & Exact solutions & approximate & & \\
\hline$S$ & $u(s)$ & $O B_{4}(s)$ & $O B_{5}(s)$ & $O B_{6}(s)$ \\
\hline 0.0 & 1.000000000000000 & 0.999979713747279 & 0.999999079504802 & 0.999999964641625 \\
\hline 0.1 & 0.904837418035960 & 0.904845662861104 & 0.904837772572798 & 0.904837426052839 \\
\hline 0.2 & 0.818730753077982 & 0.818733298758151 & 0.818730577826576 & 0.818730741637220 \\
\hline 0.3 & 0.740818220681718 & 0.740812367774209 & 0.740817962061603 & 0.740818221862245 \\
\hline 0.4 & 0.670320046035639 & 0.670314116516492 & 0.670320141390043 & 0.670320056346815 \\
\hline 0.5 & 0.606530659712633 & 0.606531291863637 & 0.606530946677938 & 0.606530659023189 \\
\hline 0.6 & 0.548811636094027 & 0.548818140965703 & 0.548811688982380 & 0.548811625685966 \\
\hline 0.7 & 0.496585303791410 & 0.496590411244172 & 0.496585024988692 & 0.496585303928115 \\
\hline 0.8 & 0.449328964117222 & 0.449325350391949 & 0.449328822447605 & 0.449328975464044 \\
\hline 0.9 & 0.406569659740599 & 0.406561706373363 & 0.406570025612431 & 0.406569650839717 \\
\hline 1 & 0.367879441171442 & 0.367899727424164 & 0.367878520676245 & 0.367879476529817 \\
\hline
\end{tabular}

TABLE 4. Relative errors of the results for Example 2.

\begin{tabular}{|c|c|c|c|c|}
\hline \multicolumn{2}{|c|}{ Points } & $e_{2}(s)$ & $e_{3}(s)$ & \\
\hline$s$ & $e_{1}(s)$ & 0.005145308871298 & $0.366073044184012 \times 10^{-3}$ & $0.202862527209913 \times 10^{-4}$ \\
\hline 0.0 & 0.051819161757163 & & & \\
\end{tabular}




\begin{tabular}{|c|c|c|c|l|}
\hline 0.1 & 0.021958238330932 & 0.000163640850578 & $0.105171097879220 \times 10^{-3}$ & $0.091119409738282 \times 10^{-4}$ \\
\hline 0.2 & 0.003695932255833 & 0.002456918271469 & $0.176615365518375 \times 10^{-3}$ & $0.031093007798068 \times 10^{-4}$ \\
\hline 0.3 & 0.023928204541512 & 0.002963404848694 & $0.035889229697377 \times 10^{-3}$ & $0.079005987509315 \times 10^{-4}$ \\
\hline 0.4 & 0.037314367701970 & 0.001764941590752 & $0.144018691863586 \times 10^{-3}$ & $0.088458031086594 \times 10^{-4}$ \\
\hline 0.5 & 0.042190610987496 & 0.000527066121947 & $0.225289914659457 \times 10^{-3}$ & $0.010422408065686 \times 10^{-4}$ \\
\hline 0.6 & 0.036618878919382 & 0.003051142572586 & $0.132548071193966 \times 10^{-3}$ & $0.118526489749161 \times 10^{-4}$ \\
\hline 0.7 & 0.018347589431006 & 0.004639112795563 & $0.111561211193742 \times 10^{-3}$ & $0.102851468277525 \times 10^{-4}$ \\
\hline 0.8 & 0.015232877209862 & 0.003749193305345 & $0.340612646760248 \times 10^{-3}$ & $0.080424934994375 \times 10^{-4}$ \\
\hline 0.9 & 0.067140584127405 & 0.001612852917474 & $0.186238077600997 \times 10^{-3}$ & $0.195621267978993 \times 10^{-4}$ \\
\hline 1 & 0.140859085770476 & 0.013986399606658 & $0.995089703894086 \times 10^{-3}$ & $0.551437521417146 \times 10^{-4}$ \\
\hline
\end{tabular}

\begin{tabular}{|c|c|c|}
\hline Points & & \\
\hline$S$ & $e_{5}(s)$ & $e_{6}(s)$ \\
\hline 0.0 & $0.092049519795356 \times 10^{-5}$ & $0.353583750056785 \times 10^{-7}$ \\
\hline 0.1 & $0.039182380274265 \times 10^{-5}$ & $0.088600215792367 \times 10^{-7}$ \\
\hline 0.2 & $0.021405255072555 \times 10^{-5}$ & $0.139737782676688 \times 10^{-7}$ \\
\hline 0.3 & $0.034910064003573 \times 10^{-5}$ & $0.015935447813295 \times 10^{-7}$ \\
\hline 0.4 & $0.014225205497485 \times 10^{-5}$ & $0.153824670545159 \times 10^{-7}$ \\
\hline 0.5 & $0.047312580242863 \times 10^{-5}$ & $0.011367008993486 \times 10^{-7}$ \\
\hline 0.6 & $0.009636886217611 \times 10^{-5}$ & $0.189647237688587 \times 10^{-7}$ \\
\hline 0.7 & $0.056143972826972 \times 10^{-5}$ & $0.002752900249466 \times 10^{-7}$ \\
\hline 0.8 & $0.031529153091736 \times 10^{-5}$ & $0.252528167292716 \times 10^{-7}$ \\
\hline 0.9 & $0.089989949626613 \times 10^{-5}$ & $0.218926370000016 \times 10^{-7}$ \\
\hline 1 & $0.250216536721577 \times 10^{-5}$ & $0.961140282617765 \times 10^{-7}$ \\
\hline
\end{tabular}




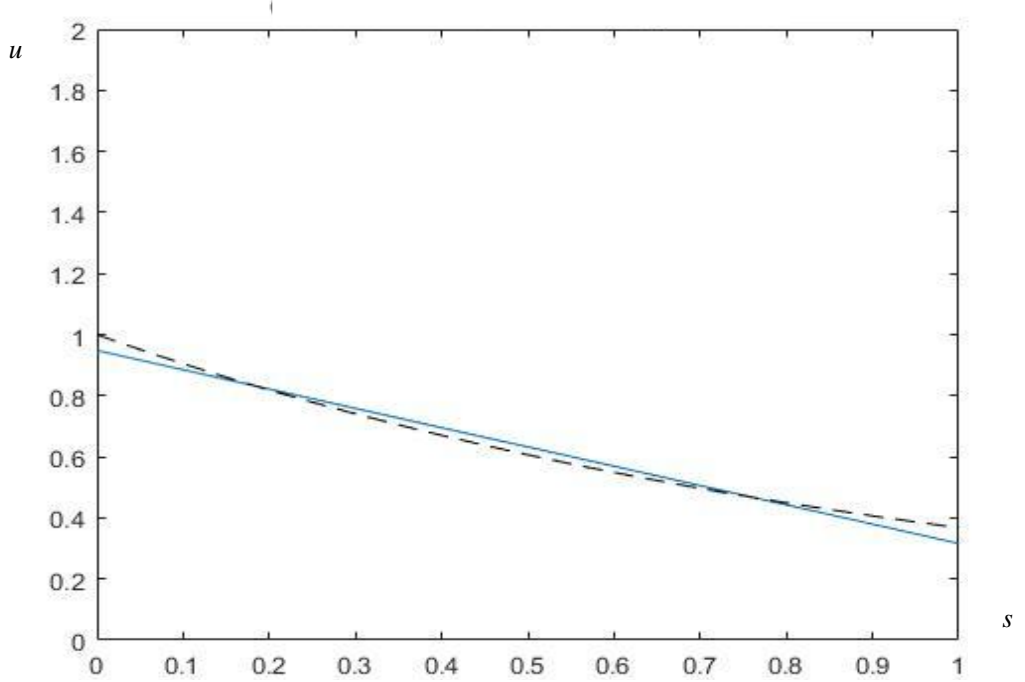

FIG. 3. Exact and approximate solutions of Example 2, for $n=1$.

Exact solution - - - -

Approximate solution

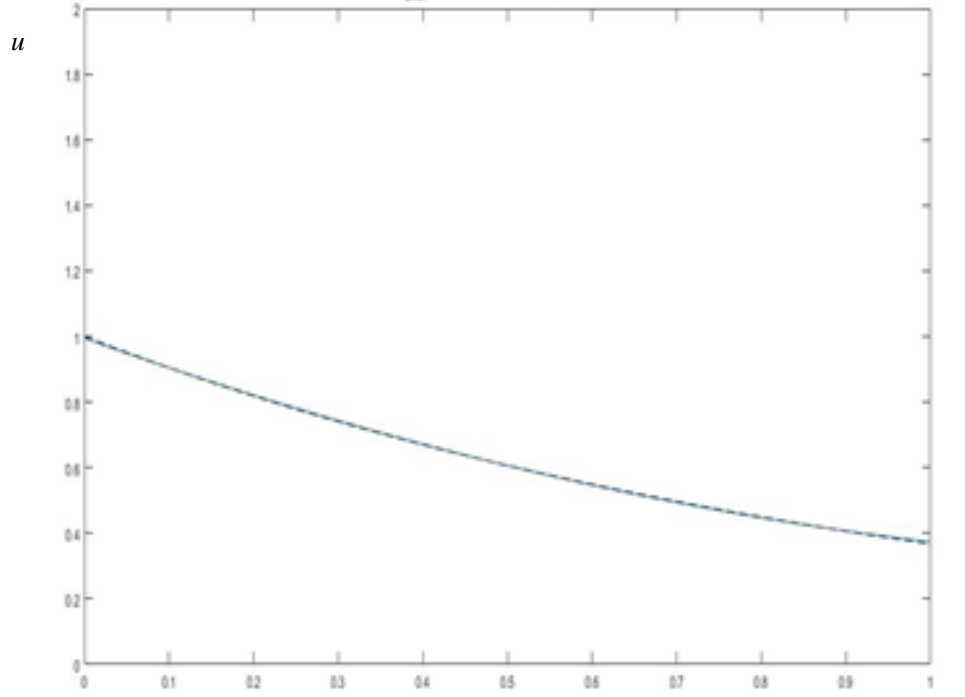

FIG. 4. Exact and approximate solutions of Example 2, for $\mathrm{n}=6$.

Exact solution - - - -

Approximate solution

Example 3. Let us the following linear Volterra integral equation [9]

$$
\mathrm{u}(\mathrm{s})=2 \sin (s)+s \sin ^{2}(s)-\int_{0}^{s} s \cos (t) u(t) d t,
$$

\section{with exact solution $\mathrm{u}(\mathrm{s})=2 \sin (s)$.}

The numerical results of this example by the proposed method are shown in Tables 5, and 6 . 
TABLE 5. Numerical results of Example 3.

\begin{tabular}{|c|c|c|c|c|}
\hline points & Exact solutions & approximate & & \\
\hline$s$ & $u(s)$ & $O B_{1}(s)$ & $O B_{2}(s)$ & $O B_{3}(s)$ \\
\hline 0.0 & 0 & 0.061181634559076 & -0.01467033533049 & -0.000497433214216 \\
\hline 0.1 & 0.199666833293656 & 0.232991812916153 & 0.198686162637868 & 0.199773406002159 \\
\hline 0.2 & 0.397338661590122 & 0.404801991273229 & 0.402672605860090 & 0.397555122325319 \\
\hline 0.3 & 0.591040413322679 & 0.576612169630305 & 0.597288994336170 & 0.591115268767070 \\
\hline 0.4 & 0.778836684617301 & 0.748422347987381 & 0.782535328066107 & 0.778721398339221 \\
\hline 0.5 & 0.958851077208406 & 0.920232526344458 & 0.958411607049901 & 0.958641064053580 \\
\hline 0.6 & 1.129284946790071 & 1.092042704701534 & 1.124917831287553 & 1.129141818921955 \\
\hline 0.7 & 1.288435374475382 & 1.263852883058610 & 1.282054000779062 & 1.288491215956153 \\
\hline 0.8 & 1.434712181799046 & 1.435663061415687 & 1.429820115524428 & 1.434956808167982 \\
\hline 0.9 & 1.566653819254967 & 1.607473239772763 & 1.568216175523652 & 1.566806148569252 \\
\hline 1 & 1.682941969615793 & 1.779283418129839 & 1.697242180776733 & 1.682306790171767 \\
\hline
\end{tabular}

\begin{tabular}{|c|c|c|c|}
\hline points & Exact solutions & approximate $s$ & \\
\hline$S$ & $u(s)$ & $O B_{4}(s)$ & $O B_{5}(s)$ \\
\hline 0.0 & 0 & 0.000058201552653 & 0.000001313510206 \\
\hline 0.1 & 0.199666833293656 & 0.199643628797039 & 0.199666304717459 \\
\hline 0.2 & 0.397338661590122 & 0.397330393233409 & 0.397338874419686 \\
\hline 0.3 & 0.591040413322679 & 0.591056329393443 & 0.591040825900263 \\
\hline 0.4 & 0.778836684617301 & 0.778853900324097 & 0.778836593494252 \\
\hline 0.5 & 0.958851077208406 & 0.958850197587599 & 0.958850632872208 \\
\hline 0.6 & 1.129284946790071 & 1.129266941261450 & 1.129284811323979 \\
\hline 0.7 & 1.288435374475382 & 1.288420479938428 & 1.288435798042511 \\
\hline 0.8 & 1.434712181799046 & 1.434721790726583 & 1.434712454407654 \\
\hline 0.9 & 1.566653819254967 & 1.566676479249239 & 1.566653224269962 \\
\hline 1 & 1.682941969615793 & 1.682884779644996 & 1.682943524234501 \\
\hline
\end{tabular}

TABLE 6. Relative errors of the results for Example 3.

\begin{tabular}{|c|c|c|c|c|}
\hline \multicolumn{5}{|l|}{ Points } \\
\hline$s$ & $e_{1}(s)$ & $e_{2}(s)$ & $e_{3}(s)$ & $e_{4}(s)$ \\
\hline 0.0 & $\infty$ & $\infty$ & $\infty$ & $\infty$ \\
\hline 0.1 & 0.166902930610839 & 0.004911535078766 & $0.533752685636406 \times 10^{-3}$ & $0.116216079727517 \times 10^{-3}$ \\
\hline
\end{tabular}




\begin{tabular}{|c|c|c|c|c|}
\hline 0.2 & 0.018783295975376 & 0.013424176365375 & $0.544776424047756 \times 10^{-3}$ & $0.020809343545582 \times 10^{-3}$ \\
\hline 0.3 & 0.024411602603047 & 0.010572172177471 & $0.126650297853899 \times 10^{-3}$ & $0.026928904361268 \times 10^{-3}$ \\
\hline 0.4 & 0.039050981072964 & 0.004748933276844 & $0.148023687580464 \times 10^{-3}$ & $0.022104386113212 \times 10^{-3}$ \\
\hline 0.5 & 0.040275859079579 & 0.000458329941897 & $0.219025831871077 \times 10^{-3}$ & $0.000917369576860 \times 10^{-3}$ \\
\hline 0.6 & 0.032978604907819 & 0.003867151080807 & $0.126742031338247 \times 10^{-3}$ & $0.015944185453058 \times 10^{-3}$ \\
\hline 0.7 & 0.019079335994466 & 0.004952808517011 & $0.043340536807069 \times 10^{-3}$ & $0.011560173873749 \times 10^{-3}$ \\
\hline 0.8 & 0.000662766810447 & 0.003409789319893 & $0.170505535562691 \times 10^{-3}$ & $0.006697460061249 \times 10^{-3}$ \\
\hline 0.9 & 0.026055162931405 & 0.000997256860120 & $0.097232274554007 \times 10^{-3}$ & $0.014463944742205 \times 10^{-3}$ \\
\hline 1 & 0.057245852948834 & 0.008497150477627 & $0.377422071285697 \times 10^{-3}$ & $0.033982140697414 \times 10^{-3}$ \\
\hline
\end{tabular}

\begin{tabular}{|c|c|}
\hline Point & Relative error \\
\hline$S$ & $e_{5}(s)$ \\
\hline 0.0 & $\infty$ \\
\hline 0.1 & $0.264729093103320 \times 10^{-5}$ \\
\hline 0.2 & $0.053563769298534 \times 10^{-5}$ \\
\hline 0.3 & $0.069805308517978 \times 10^{-5}$ \\
\hline 0.4 & $0.011699891748172 \times 10^{-5}$ \\
\hline 0.5 & $0.046340480666859 \times 10^{-5}$ \\
\hline 0.6 & $0.011995740515675 \times 10^{-5}$ \\
\hline 0.7 & $0.032874534285355 \times 10^{-5}$ \\
\hline 0.8 & $0.019000926552937 \times 10^{-5}$ \\
\hline 0.9 & $0.037978077717600 \times 10^{-5}$ \\
\hline 1 & $0.092375063196229 \times 10^{-5}$ \\
\hline
\end{tabular}




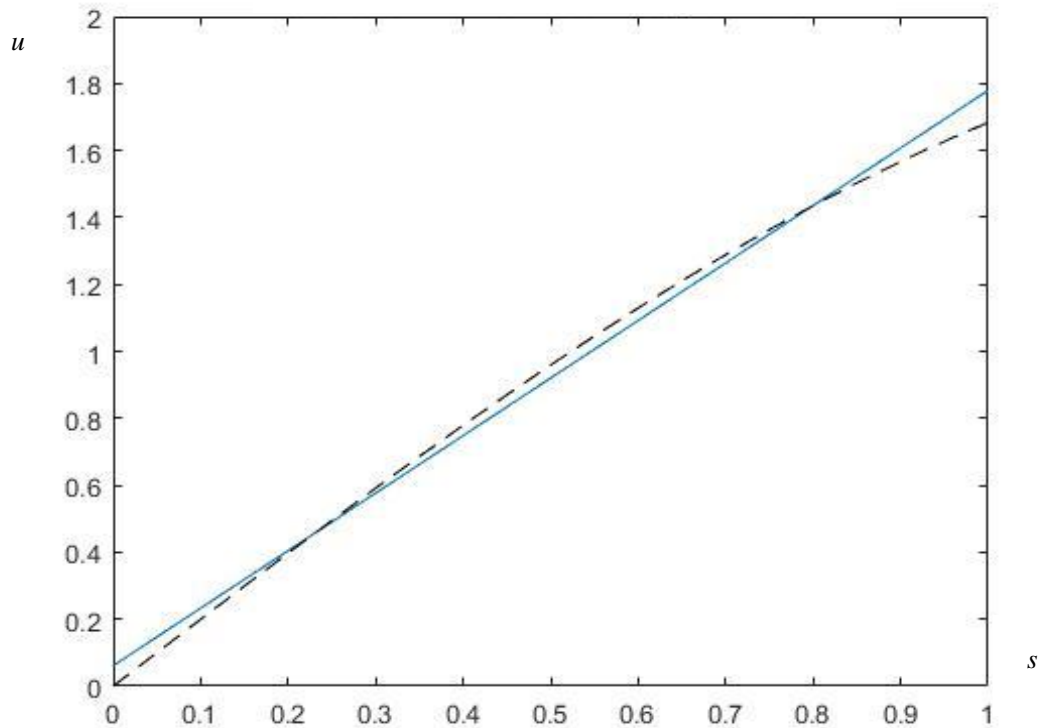

FIG. 5. Exact and approximate solutions of Example 3, for $n=1$.

Exact solution - - - -

Approximate solution

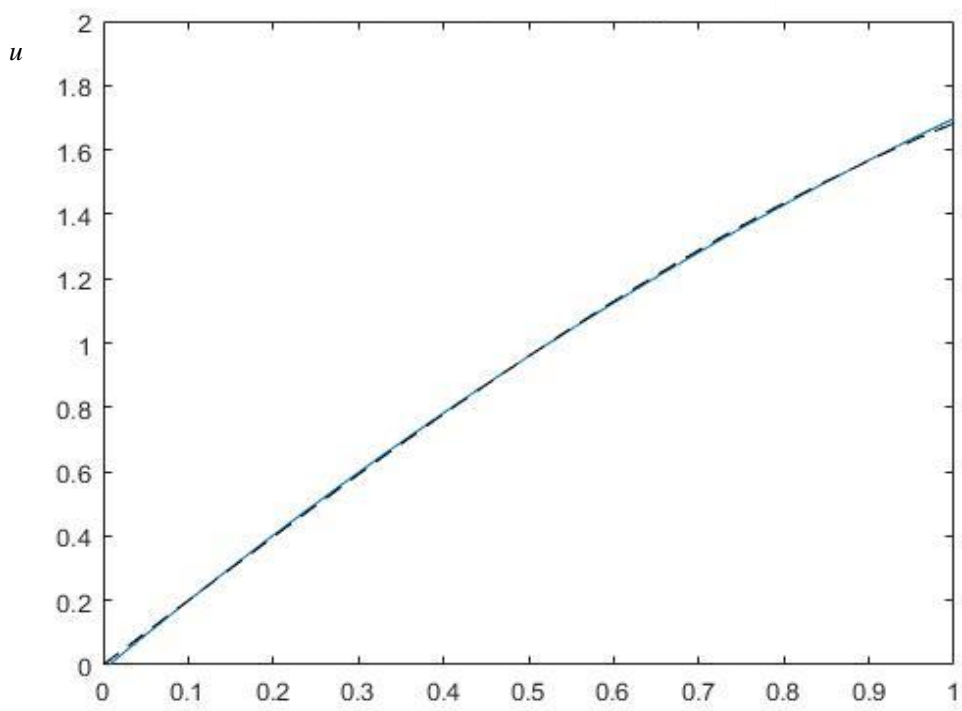

FIG. 6. Exact and approximate solutions of Example 3, for $n=3$.

Exact solution - - - -

Approximate solution

Example 4. Consider the following linear Volterra integral equation

$$
\mathrm{u}(\mathrm{s})=\left(\frac{4 \cos (s)+\cos (2 s)-1}{4}\right)+\int_{0}^{s} \sin (t) u(t) d t,
$$

\section{with exact solution $\mathrm{u}(\mathrm{s})=\cos (s)$.}

Numerical results of this example by the proposed method are reported in Tables 7, and 8 . 
TABLE 7. Numerical results of Example 4.

\begin{tabular}{|c|c|c|c|c|}
\hline points & Exact solutions & approximat & & \\
\hline$S$ & $u(s)$ & $O B_{1}(s)$ & $O B_{2}(s)$ & $O B_{3}(s)$ \\
\hline 0.0 & 1.000000000000000 & 1.076762136252093 & 1.003477391185374 & 0.999463036153306 \\
\hline 0.1 & 0.995004165278026 & 1.029156699292469 & 0.995448166565130 & 0.995132855257258 \\
\hline 0.2 & 0.980066577841242 & 0.981551262332844 & 0.978816238355181 & 0.980285501366648 \\
\hline 0.3 & 0.955336489125606 & 0.933945825373220 & 0.953581606555526 & 0.955395415444817 \\
\hline 0.4 & 0.921060994002885 & 0.886340388413595 & 0.919744271166166 & 0.920937038455103 \\
\hline 0.5 & 0.877582561890373 & 0.838734951453971 & 0.877304232187100 & 0.877384811360847 \\
\hline 0.6 & 0.825335614909678 & 0.791129514494346 & 0.826261489618328 & 0.825213175125389 \\
\hline 0.7 & 0.764842187284489 & 0.743524077534722 & 0.766616043459851 & 0.764896570712067 \\
\hline 0.8 & 0.696706709347165 & 0.695918640575097 & 0.698367893711669 & 0.696909439084222 \\
\hline 0.9 & 0.621609968270664 & 0.648313203615473 & 0.621517040373781 & 0.621726221205193 \\
\hline 1 & 0.540302305868140 & 0.600707766655848 & 0.536063483446187 & 0.539821358038321 \\
\hline
\end{tabular}

\begin{tabular}{|c|c|c|}
\hline points & Exact solutions & approximate solutions \\
\hline$S$ & $u(s)$ & $O B_{4}(s)$ \\
\hline 0.0 & 1.000000000000000 & 0.999985459450244 \\
\hline 0.1 & 0.995004165278026 & 0.995010021149647 \\
\hline 0.2 & 0.980066577841242 & 0.980069291457862 \\
\hline 0.3 & 0.955336489125606 & 0.955332582356750 \\
\hline 0.4 & 0.921060994002885 & 0.921055912493191 \\
\hline 0.5 & 0.877582561890373 & 0.877582007179078 \\
\hline 0.6 & 0.825335614909678 & 0.825340298391323 \\
\hline 0.7 & 0.764842187284489 & 0.764846924771851 \\
\hline 0.8 & 0.696706709347165 & 0.696704731627604 \\
\hline 0.9 & 0.621609968270664 & 0.621603270930538 \\
\hline 1 & 0.540302305868140 & 0.540318801317627 \\
\hline
\end{tabular}

TABLE 8. Relative errors of the results for Example 4.

\begin{tabular}{|c|c|c|c|c|}
\hline \multicolumn{3}{|l|}{ Points } & $e_{2}(s)$ & $e_{3}(s)$ \\
\hline$s$ & $e_{1}(s)$ & 0.003477391185374 & $0.536963846694438 \times 10^{-3}$ & $0.145405497559148 \times 10^{-4}$ \\
\hline 0.0 & 0.076762136252093 & & \\
\hline
\end{tabular}




\begin{tabular}{|c|c|c|c|c|}
\hline 0.1 & 0.034324011100898 & 0.000446230581337 & $0.129336121116557 \times 10^{-3}$ & $0.058852734752945 \times 10^{-4}$ \\
\hline 0.2 & 0.001514881259264 & 0.001275769946992 & $0.223376177043643 \times 10^{-3}$ & $0.027688084476281 \times 10^{-4}$ \\
\hline 0.3 & 0.022390711540773 & 0.001836926140742 & $0.061681219006516 \times 10^{-3}$ & $0.040894165570653 \times 10^{-4}$ \\
\hline 0.4 & 0.037696315244440 & 0.001429571814780 & $0.134579087149462 \times 10^{-3}$ & $0.055170175782803 \times 10^{-4}$ \\
\hline 0.5 & 0.044266616183350 & 0.000317155006674 & $0.225335527519557 \times 10^{-3}$ & $0.006320901513218 \times 10^{-4}$ \\
\hline 0.6 & 0.041445079792268 & 0.001121816012691 & $0.148351509468203 \times 10^{-3}$ & $0.056746389716564 \times 10^{-4}$ \\
\hline 0.7 & 0.027872559992349 & 0.002319244681914 & $0.071104115963369 \times 10^{-3}$ & $0.061940717204280 \times 10^{-4}$ \\
\hline 0.8 & 0.001131134179555 & 0.002384338118489 & $0.290982897590362 \times 10^{-3}$ & $0.028386687468770 \times 10^{-4}$ \\
\hline 0.9 & 0.042958183922143 & 0.000149495506229 & $0.187019096318854 \times 10^{-3}$ & $0.107741839229423 \times 10^{-4}$ \\
\hline 1 & 0.111799376259650 & 0.007845279163008 & $0.890145802813948 \times 10^{-3}$ & $0.305300371807670 \times 10^{-4}$ \\
\hline
\end{tabular}

$u$

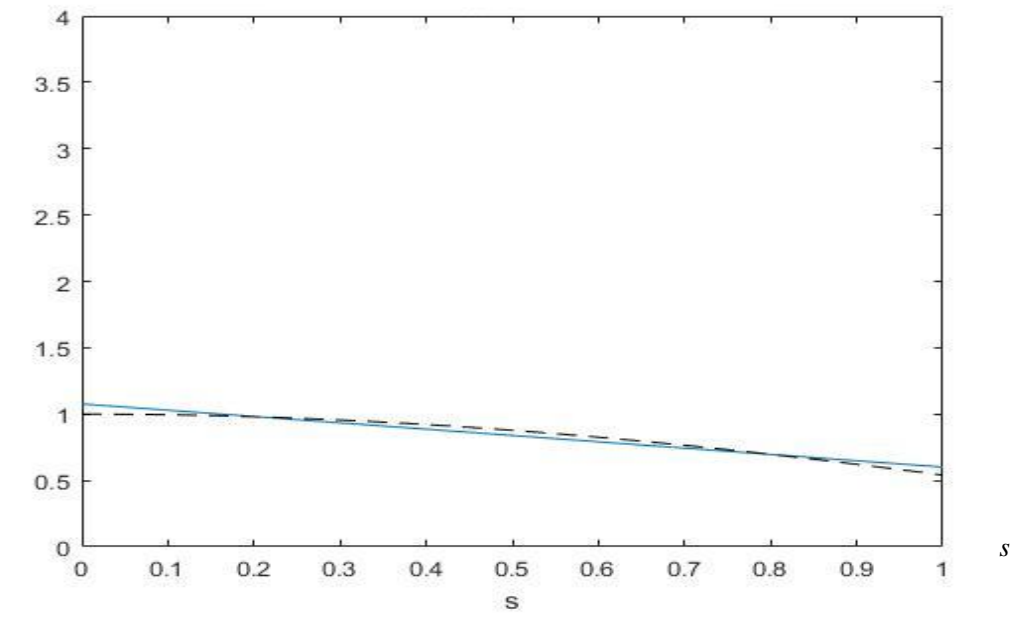

FIG. 7. Exact and approximate solutions of Example 4, for $n=1$.

Exact solution - - . -

Approximate solution

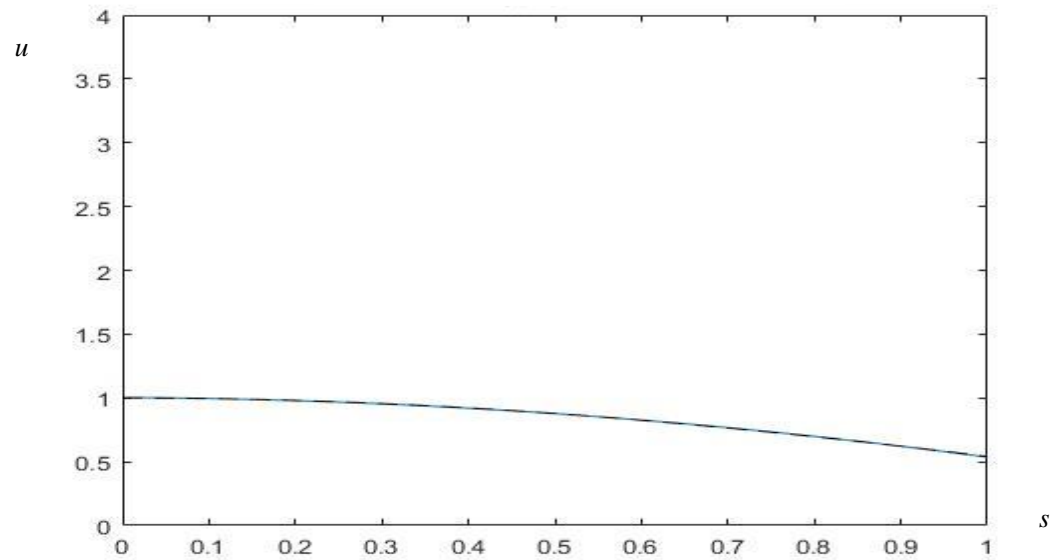

FIG. 8. Exact and approximate solutions of Example 4, for $n=2$. 


\section{Conclusion}

The results of this study showed that this method is very accurate for linear Volterra integral equations of the second kind. The important thing is that as the degree of the orthonormal Bernstein polynomials increase the accuracy of approximate solution also will increase. Moreover, by examining the relative error, we conclude that, as the degree of polynomials is raised the relative error is decreased. It should be noted that its computational cost is high. By making some changes, this method can be used for solving two-dimensional linear Volterra integral equations.

\section{Acknowledgement}

We want to thank the anonymous referees for the careful reading of our article and for giving constructive feedback that can significantly help to improve the quality of the article.

\section{References}

[1] Bernstein, S. "Démo istration du th'eorème de Weierstrass fondée sur le calcul des probabilités." Communication of the Kharkov Mathematical Society 13.1 (1912): 1-2.

[2] Biazar, J., and Eslami, M. "Homotopy perturbation and Taylor series for Volterra integral equations of the second kind." Middle-East Journal of Scientific Research 7.4 (2011): 604-609.

[3] Biazar, J., \& Ghazvini, H. "He's homotopy perturbation method for solving systems of Volterra integral equations of the second kind." Chaos, Soli tons \& Fractals 39.2 (2009): 770-777.

[4] Bustamante, J. Bernstein operators and their properties. Springer International Publishing, 2017.

[5] Farin, G. E., and Farin, G. Curves and surfaces for CAGD: a practical guide. Morgan Kaufmann, 2002.

[6] Jackson, D. Fourier series and orthogonal polynomials. Courier Corporation, 2012.

[7] Khan, F., et al. "Numerical approach based on Bernstein polynomials for solving mixed Volterra- Fredholm integral equations." AIP Advances 7.12 (2017): 125123-3.

[8] L.M. Delves, J.L. Mohamed, Computational Methods for Integral Equations, Cambridge University Press, Cambridge, 1985.

[9] Ramadan, M. A., \& Ali, M. R. "Numerical solution of Volterra-Fredholm integral equations using hybrid orthonormal bernstein and block-pulse functions." Asian Research Journal of Mathematics (2017): 1-14. 